\title{
Application of Adaptive Fuzzy Controllers for the Automation of Medical Devices
}

\author{
Alejandro Mendoza García, \\ Nicole Sprunk, \\ Benedikt Baumgartner \\ and Alois Knoll \\ Insititute of Robotics and Embedded Systems \\ Technische Universität München \\ Munich,Germany
}

\author{
Ulrich Schreiber, \\ Stefan Eichhorn, \\ Markus Krane \\ and Rüdiger Lange \\ Department of Experimental Surgery \\ Deutsches Herzzentrum München \\ Munich, Germany
}

\begin{abstract}
This paper describes an adaptive fuzzy control algorithm that may be used for the automation of medical devices. The automation system consists of a set of controllers containing the expertise of the physician and a main fuzzy controller that creates new rules based on this knowledge plus additional information about the patient. An adaptive mechanism evaluates the performance of the controller by using reference models and modifies its rules when needed. As a test case the automation of an extra-corporeal support system is presented, where a centrifugal pump speed is adjusted based on desired pressures and flow values.
\end{abstract}

\section{INTRODUCTION}

Current medical devices have advanced sensoring systems capable of constantly monitoring patients and helping medical doctors in giving better treatment to patients. With the increase of computational power, miniaturization and decreased power consumption, these devices may be easily taken along side the patient. Going one step further these devices may directly provide patient treatment with the integration of intelligent systems.

For these devices to be accepted in the medical field they should be capable of taking decisions similar to experienced practitioners. Making medical decisions is not an easy task. Decisions are based on previous knowledge, experience and observation of the patient. The device should be able to work together with the physician, and he should know why certain decisions were taken. Fuzzy logic provides a simple way to transfer that knowledge and experience to the automation system by describing how the system should behave with the creation of rules. Additionally the controller decisions may be easily understood by the operator.

When treating patients with different preconditions it is possible that they will respond differently for the same kind of treatment. For this reason adaptive mechanisms are needed, capable of observing the reactions of the patient and modifying the rule base to improve the given treatment.

The decisions taken by physicians may depend upon the observation of various signals. Creating a single rule base that contains this knowledge including all of the possible input signals with the combination of its sets may become a highly complex task if not impossible and may need considerably large number of rules. We solve this problem by subdividing the rule base into several smaller controllers. This gives the possibility of describing the controller behavior focusing on specific parameters, using less number of inputs for each controller. The output of these controllers are added to create a single output. An adaptive fuzzy controller dynamically creates its own rules using the information of the smaller controllers. This controller is capable of modifying its rules by observing the response of the patient in comparison to what is expected.

Previous studies in our department involved the use of fuzzy logic as a control strategy for an extra-corporeal support system [1]. The adaptive mechanism was introduced into this system and in-vitro studies were used to provide the results of this paper.

\section{FUZZY LOGIC}

The basic configuration of a fuzzy logic system shown in figure 1 is based on a fuzzification block gathering all the inputs of the system and its corresponding sets, a rule-base, an inference mechanism and defuzzification. To this configuration additional scaling factors were introduced to the inputs $\left(g_{n}\right)$ and output $\left(h_{0}\right)$. This allows the easy configuration of the range of values covered by the fuzzy sets. The fuzzy sets of the input and output variables are of a triangular or trapezoid shape and are arranged to cover the complete universe of disclosure, which from the fuzzy controller perspective corresponds from -1 to +1 and is converted to the outside world with its corresponding gain values. The inputs may correspond to errors between targets and values $\left(e_{i}\right)$, the change of error $\left(d e_{i}\right)$ or current values $\left(v_{i}\right)$.

Fuzzy sets are expressed in linguistic terms such as "Low" or "Very Low" that may be easily understood. However when the number of sets is increased determining the correct names for each set may not be simple. For this reason the naming of sets was changed from linguistic terms to signed numbers as shown in table I making it simple to extend to $n$ number of positive and negative fuzzy sets. It is clear however that “-2" for example does not refer to the value itself but to the 


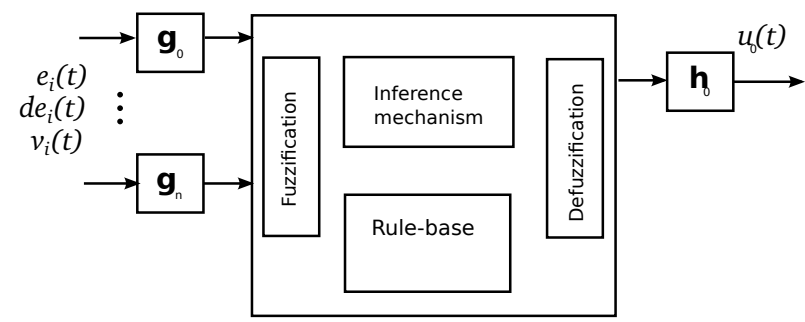

Fig. 1. Fuzzy Controller architecture

second set on the negative side in the universe of disclosure of a particular variable.

\begin{tabular}{|c|c|c|}
\hline Input & Output & Equivalent \\
\hline "Very Low" & "Big Decrease" & -2 \\
"Slightly Low" & "Small Decrease" & -1 \\
"Normal" & "Steady" & 0 \\
"Slightly High" & "Small Increase" & +1 \\
"Very High" & "Big Increase" & +2 \\
\hline
\end{tabular}

TABLE I

REPRESENTATION OF INPUTS AND OUTPUTS

\section{A. Reference Models}

Reference models are used to describe how a specific input is expected to reach its given target over time. When the controller is activated the reference signals are set to the current value of each parameter. A second order function is used as a reference signal with a specified stabilizing time. With the target value, the reference model and the current signal value two error signals are generated: a target error $(T e)$ which refers to the difference between the current value of the input signal and the target; and a reference error $(R e)$ which refers to the difference between the current value of the input signal and the reference model being calculated.This is depicted in figure 2.

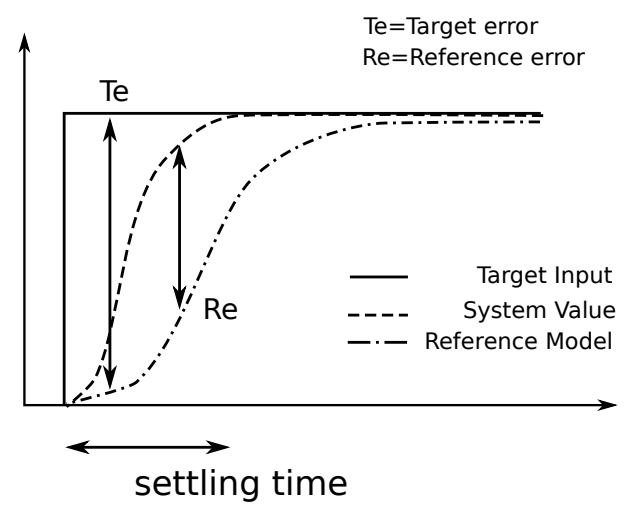

Fig. 2. Reference model and generated errors.

\section{KNOWLEDGE BASE}

When dealing with multiple inputs and defining several sets for each input, the creation of a single rule base containing all the possible combinations may not be an easy task. This was resolved by defining smaller controllers containing rules of how the controller should react to specific inputs. The result of all of these controllers is then added to form a single output signal. Predefined rule structures can be used to easily construct these smaller controllers, for example a Fuzzy-PI type of controller may be used for one input, using its error and change of error. The rule structure for this type of controller is shown in table II and is further described by $\mathrm{Li}$ [2].

\begin{tabular}{|c||c|c|c|c|c|c|c|}
\hline$\dot{e} / \mathrm{e}$ & -3 & -2 & -1 & 0 & +1 & +2 & +3 \\
\hline \hline-3 & +6 & +5 & +4 & +3 & +2 & +1 & 0 \\
-2 & +5 & +4 & +3 & +2 & +1 & 0 & -1 \\
-1 & +4 & +3 & +2 & +1 & 0 & -1 & -2 \\
0 & +3 & +2 & +1 & 0 & -1 & -2 & -3 \\
+1 & +2 & +1 & 0 & -1 & -2 & -3 & -4 \\
+2 & +1 & 0 & -1 & -2 & -3 & -4 & -5 \\
+3 & 0 & -1 & -2 & -3 & -4 & -5 & -6 \\
\hline \multicolumn{1}{c|}{ TABLE II } \\
RULE BASE OF A FUZZY-PI CONTROLLER
\end{tabular}

\section{ADAPTING FUZZY CONTROLlER}

At the beginning the previously defined fuzzy controllers will not be optimally tuned for all the possible scenarios and specific patient reactions. Therefore an adaptive mechanism is needed capable of recognizing when a change must be made and how the rules should be modified. It is difficult to detect how the rules should be changed in each small controller, this was solved by introducing an empty fuzzy controller that is capable of creating its own rules. This fuzzy controller uses the same inputs as the knowledge base controllers together with additional inputs that help to characterize a specific situation. This part is called online rule learning. The second part is the adaptive mechanism where a function is used to grade the performance of the active rules and modify them when required.

\section{A. Online Rule Learning}

The online learning mechanism is described in figure 3. When no rule exists for the current inputs with a degree of truth higher than a predefined threshold a new rule is created. For the creation of this new rule the input sets with the highest degree of truth for each input is used as antecedents; for the selection of the output set the knowledge base controllers are used to obtain a reference value, and with this value the set with highest degree of truth is used as a consequent.

We can observe from figure 3 that when the new rule is created there can be a slight difference between the output obtained from the base controllers and the output obtained from the new rule. As an example the reference input is -1.3 , the learning algorithm will then select the output set with that gives the maximum degree of truth, which in this case is " -1 ". If this is the only existing rule then the output of the controller will be -1 . This difference can be reduced by increasing the number of sets used for each variable in the adaptive controller. 


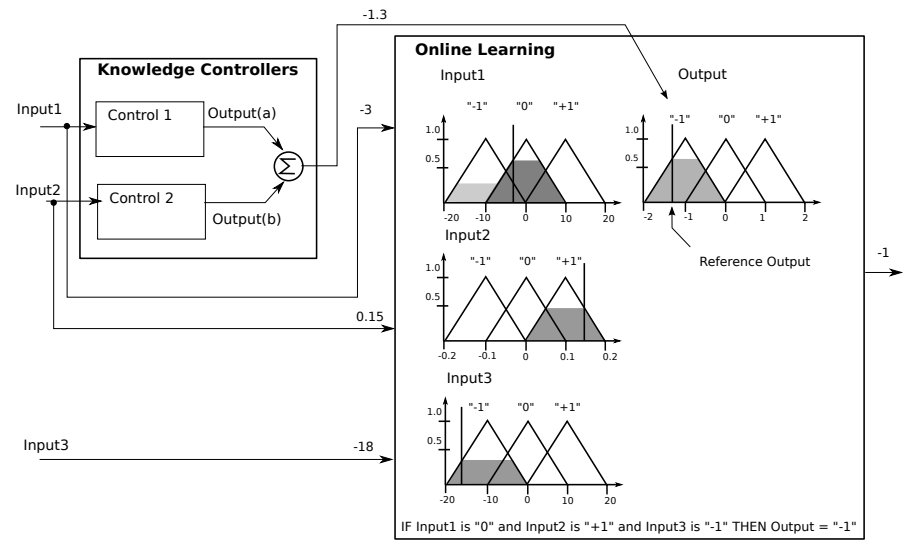

Fig. 3. Online rule learning.

\section{B. Adapting Algorithm}

The adaptive mechanism shown in figure 4 consists of the main controller containing the previously learned rules and a Fuzzy Inverse Model (FIM). The FIM is a fuzzy controller that has the total normalized reference error $(N R E)$ and its derivative as input. The $N R E$ is calculated as follows:

$$
\begin{array}{r}
\eta R e_{i}=\frac{R e_{i}}{T_{i}-v_{\min , i}} \\
N R E=\frac{\sum_{i=1}^{n} \eta R e_{i} \cdot w_{i}}{\sum_{i=1}^{n} w_{i}}
\end{array}
$$

Where $\eta R e_{i}$ is the normalized reference error; $R e_{i}$ corresponds to the reference error; $T_{i}$ is the target and $v_{\min , i}$ is the minimum expected value of var $i . N R E$ adds all of the normalized reference errors each multiplied by their individual weights $w_{i}$ and divided by the sum of all the parameter weights. This normalization allows the possibility of using input signals of different nature such as pressure and flow and still be able to compare in an equitative manner how far are the input signals from their corresponding reference model.

The rule base of the FIM is configured as a Fuzzy-PI as described in table II. The FIM monitors how different the current system is from the reference model and if this difference is increasing or decreasing. The created rules in the main fuzzy controller are modified by the adapting algorithm according to the following steps:

1) For each created rule from the learning algorithm a weight is assigned, starting with a value of 0 .

2) The rules active with a degree of truth higher than a given threshold in each step are saved in a vector.

3) The NRE and its derivative are calculated

4) After a predefined delay has passed the $N R E$ and its change over time is used as input for the FIM. The FIM then generates the signal $p(t)$.

5) For the rules active at time - delay the rule weight is added with $p(t)$ multiplied by its saved rule truth $T r$.

$$
w_{r, i}=w_{r, i}+p(t) \cdot T r_{i}
$$

6) Rules are modified in the adaptive controller; if the weight of a rule exceeds +1 then the output set of the current rule is modified to use the next higher continuous set. If the rule weight is lower or equal to - 1 the output set is changed to the next lower continuous set. When the rule is changed the rule weight $w_{r, i}$ is set back to 0 .

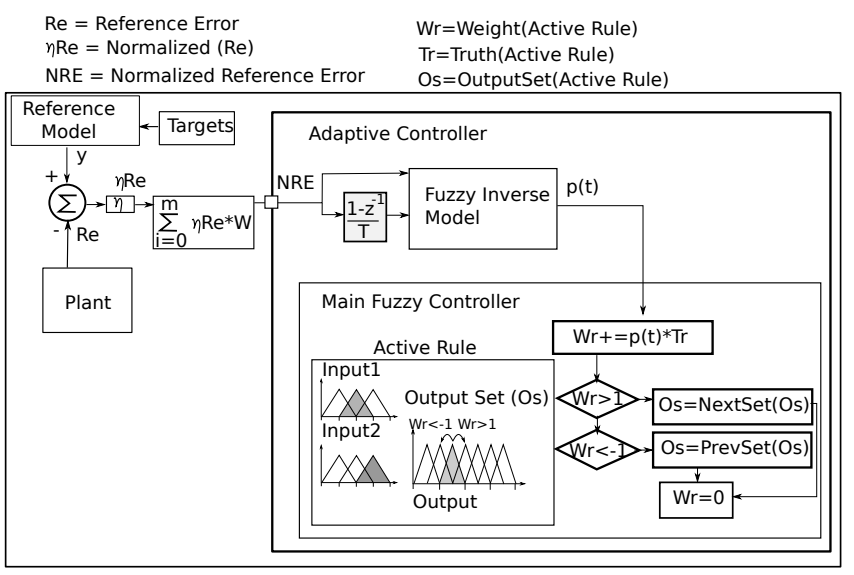

Fig. 4. Adaptive algorithm.

\section{TEST CASE}

This adaptive fuzzy controller is currently being integrated for the automation of a portable extra-corporeal support system (ECSS) [1], [3]-[5]. This device is intended to be used with patients that suffer from cardiac arrest outside the hospital. The controller should be capable of automatically adjusting the device parameters such as pump speed to obtain proper values of pressure and blood flow. An in-vitro system was created to test different control mechanisms [4]. The system consists of a centrifugal pump, two adjustable resistance, used to simulate the systemic vascular resistance and system components, and a compliance.

\section{A. Implementation}

Previous work included the development of an acquisition system for medical signals [6] additionally a program was designed to easily implement the designed controller [7]. This program was used in this work obtaining the control structure shown in figure 5. The speed control of the centrifugal pump in the ECSS is established depending on the extra-corporeal flow rate (EFR) and mean arterial pressure (MAP).

Two Fuzzy-PI type of controllers were used as knowledge base to define the control behavior for MAP and EFR. The input values are obtained from an analog to digital converter(ADC). The output is defined as an increase or decrease of speed, converted to voltage with a digital to analog converter (DAC). A reference model was used for each signal. The reference error of each signal and the change over time was introduced into the Fuzzy-PI controllers integrating the knowledge base. These same signals and the target errors were introduced into the adaptive controller. All the inputs of this controller were defined with 7 input sets and the output was defined with 21 output sets.

The threshold for the learning mechanism was set considering the overlap of the input sets. If a threshold is chosen too 


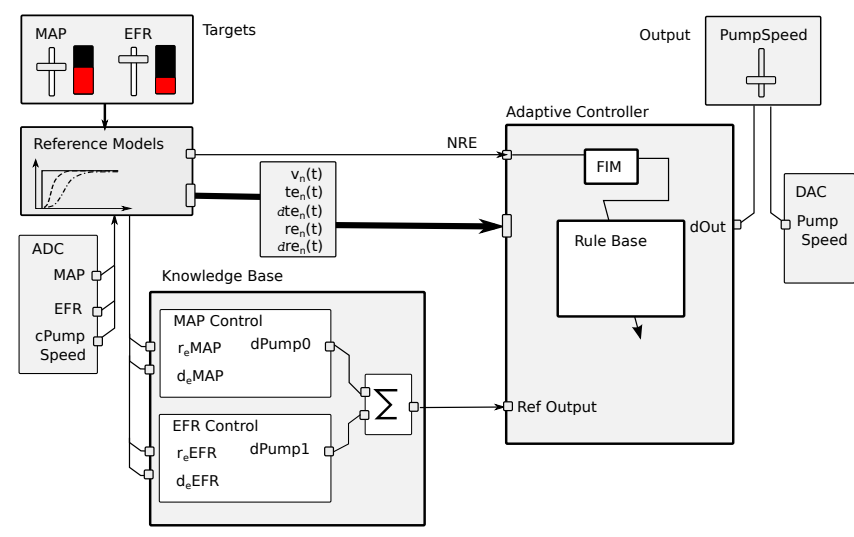

Fig. 5. Adaptive Fuzzy Controller of pressure and flow.

high (0.9) and there is a small overlap between sets then more rules will be created, with the risk of creating repeated rules. If on the other hand the threshold is too low $(0.5)$ then less rules will be created, ignoring valuable information from the knowledge controllers. With this considerations a threshold of 0.7 was selected and the input sets were set to overlap $45 \%$ of the neighbor set. The delay for the adaptive mechanism was selected according to the activation times of the controller and the dynamics of the system. The activation time of the controller was $100 \mathrm{~ms}$. The stabilization time of the reference model was set to 5 seconds. With this consideration the delay was set to half a second, storing a total of 5 active rule sets.

\section{B. Results}

Four control configurations were tested. The first one is the result of using directly the output of the knowledge base. The second controller refers to Learning. This controller starts with an empty rule base and creates the required rules using the knowledge base controllers. The third control is Adapting. For this case the knowledge base controllers are not activated and the reference output is always set to zero. The adaptive mechanism is activated and every time a new rule is created with new inputs the output set is started as the " 0 " set and is then modified according to the adaptive algorithm described previously, using the $N R E$. The last results correspond to $L+A$, for this controller the learning and adaptive mechanisms were both activated.

A mean absolute error (MAE) is used to calculate the performance of each controller, this is done using the absolute sum of the normalized reference errors multiplied by the weight of input $i$ as shown in equation 4 .

$$
M A E=\sum_{i=0}^{n}\left\|\eta R e_{i} \cdot w_{i}\right\|
$$

Figure 6 shows the results of setting the controller to different flow rates and pressure levels. The first and second graph show the control values of pressure and flow correspondingly. This also includes the target value signal and the signal generated by the reference model. The third graph shows the
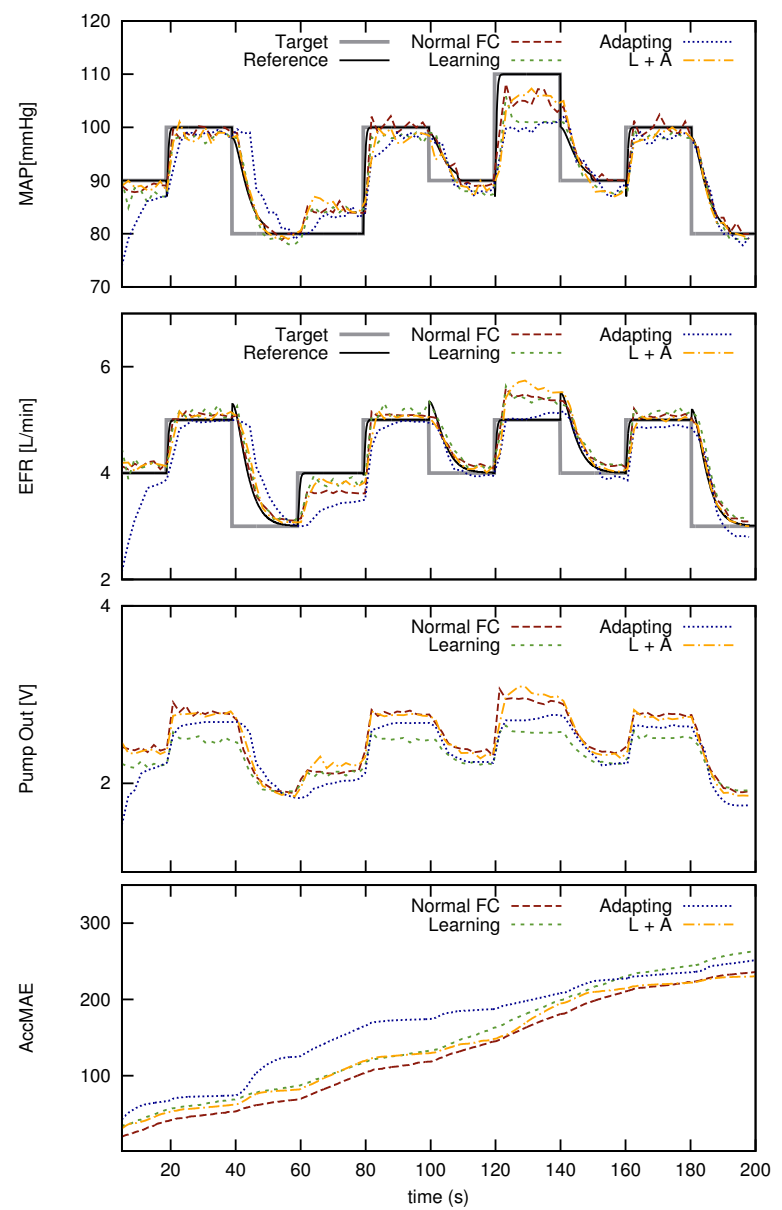

Fig. 6. Control results for different control algorithms.

pump output. The last graph shows the accumulative MAE to compare different control mechanisms.

\begin{tabular}{|c||c|c|c|c|}
\hline & Normal FC & Learning & Adapting & $\mathrm{L}+\mathrm{A}$ \\
\hline AvgMAE & 0.121 & 0.130 & 0.127 & 0.11 \\
\hline
\end{tabular}

TABLE III

COMPARISON OF AVERAGE MAE CALCULATED BETWEEN EACH CONTROL ALGORITHM

Table III shows the average MAE for the different control algorithms.

What may be observed in these results in respect to reaching the desired target values is that the exact target pressure (MAP) and flow (EFR) may not always be achieved due to their dependency with each other. The controller will try to reach an equilibrium point of both inputs. In terms of control performance the results of the MAE from the learning algorithm were slightly higher than the Normal FC. The adaptive controller had the worst response over the first 160 seconds since it does not have previous knowledge about how to control the system, however as the system corrects itself with the adaptive mechanism it is able to improve the results compared to the learning algorithm. The $L+A$ controller 
started similar to the learning algorithm, however due to the adaptive mechanism it is able to improve performance and after a certain time outperform the Normal FC.

The number of rules created by the learning algorithm were 112. For the adaptive mechanism 140 rules were created and for the $L+A 121$.

Considering the number of inputs for the adaptive controller (4), with the number of sets for each input (7) the number of rules needed to cover all the possible combinations would have been more than 2000 rules, the manual creation of this rules would represent a great challenge and could affect the performance of the controller, however with the proposed mechanism only the needed rules are created with help from the knowledge controller, without having a significant decrease in performance and allowing a fine adjustment by the adaptive mechanism over inputs with a higher number of sets.

Further improvements are still possible to make the adaptive mechanism more efficient. If the dynamics of the system is unknown and the delay of the adaptive mechanism is fixed some oscillations may be generated once the controller has reached a certain state. An alternative is to switch off the adaptive mechanism after a certain performance has been achieved. A second approach is to make the delay dynamically change according to the response of the system.

\section{CONCLUSION}

Medical devices may help in providing better attention to patients with the integration of automation systems that, based on what is monitored from the patient, may react and give proper treatment. By using fuzzy logic it was possible to integrate the knowledge and experience of the physiologist in the form of rules, providing in a similar manner a feedback to the operator of why certain actions were taken. An adaptive system may also provide a way to improve the performance of the controller by using the information acquired through the specialist and monitoring the response of the patient.

The presented adaptive algorithm was able to control the system even if it did not have previous knowledge on how to control the system, however to assure best treatment for the patient the system should start with previous knowledge of the patient and be able to learn and adapt as quickly as possible.

\section{ACKNOWLEDGMENT}

This work was supported by an unrestricted grant from Bayerische Forschungsstiftung.

\section{REFERENCES}

[1] B. Baumgartner, A. Mendoza Garcia, U. Schreiber, S. Eichhorn, M. Krane, R. Bauernschmitt, and A. Knoll, "A comprehensive approach towards extra-corporal circulation control using fuzzy logic," Fuzzy Systems (FUZZ), 2010 IEEE International Conference on, pp. 1 -5, july 2010.

[2] H.-X. Li, "Approximate model reference adaptive mechanism for nominal gain design of fuzzy control system," Systems, Man, and Cybernetics, Part B: Cybernetics, IEEE Transactions on, vol. 29, no. 1, pp. $41-46$, feb 1999.

[3] A. Mendoza G, B. Baumgartner, U. Schreiber, S. Eichhorn, M. Krane, R. Bauernschmitt, and A. Knoll, "Design of a fuzzy controller for the automation of an extracorporeal support system with the use of a simulation environment," pp. 6698 -6701, 31 2010-sept. 42010.
[4] U. Schreiber, S. Eichhorn, A. Mendoza, B. Baumgartner, R. Bauernschmitt, R. Lange, A. Knoll, and M. Krane, "A new fuzzy controlled extracorporeal circulation system. first results of an in-vitro investigation," Computers in Cardiology, pp. 497-500, 2009.

[5] M. Krane, D. Mazzitelli, U. Schreiber, A. M. Garcia, B. Voss, C. Badiu, R. Lange, and R. Bauernschmitt, "First experience with a new portable cardiopulmonary bypass system - lifebridge b2t with percutaneous femoral cannulation," Comp. In Cardiology, pp. 269-272, 2008.

[6] A. Mendoza Garcia, B. Baumgartner, U. Schreiber, M. Krane, A. Knoll, and R. Bauernschmitt, "Automedic: Fuzzy control development platform for a mobile heartlung machine." IFMBE Proceedings, vol. 25(7), pp. 685-688, 2009.

[7] A. Mendoza G, M. Rodriguez H, B. Baumgartner, U. Schreiber, and A. Knoll, "Embedded platform for automation of medical devices," Computers in Cardiology, 2011.

[8] J. H. T. Bates and M. P. Young, "Applying fuzzy logic to medical decision making in the intensive care unit," Am. J. Respir. Crit. Care Med., vol. 167, no. 7, pp. 948-952, 2003.

[9] C. W. Chan, K. C. Cheung, and W. K. Yeung, "A computation-efficient on-line training algorithm for neurofuzzy networks." Int. J. System Science, pp. 297-306, 2000.

[10] H.-X. Li and H. Gatland, "Conventional fuzzy control and its enhancement," Systems, Man, and Cybernetics, Part B: Cybernetics, IEEE Transactions on, vol. 26, no. 5, pp. $791-797$, oct 1996.

[11] D. G. Mason, D. A. Linkens, and N. D. Edwards, "Self-learning fuzzy logic control in medicine," in Proceedings of the 6th Conference on Artificial Intelligence in Medicine in Europe, ser. AIME '97. London, UK: Springer-Verlag, 1997, pp. 300-303.

[12] T. Nazmy, H. El-Messiry, and B. Al-Bokhity, "Adaptive neuro-fuzzy inference system for classification of ecg signals," in Informatics and Systems (INFOS), 2010 The 7th International Conference on, march 2010, pp. $1-6$.

[13] A. Nurnberger, D. Nauck, and R. Kruse, "Neuro-fuzzy control based on the nefcon-model under matlab/simulink," SOFT COMPUTING, vol. 2, pp. 168-182, 1997.

[14] M. Suzer and K.-D. Kang, "Adaptive fuzzy control for utilization management," in Object Oriented Real-Time Distributed Computing (ISORC), 2008 11th IEEE International Symposium on, may 2008, pp. $383-390$.

[15] W.-M. Lippe and S. Niendieck, "Different methods for the fineoptimization of fuzzy-rule-based-systems," in Fuzzy Systems, 2002. FUZZ-IEEE'02. Proceedings of the 2002 IEEE International Conference on, vol. 2, 2002, pp. $1210-1215$.

[16] C. Koutsojannis and I. Hatzilygeroudis, "Using a neurofuzzy approach in a medical application.” in KES (2)'07, 2007, pp. 477-484.

[17] B. Misgeld, J. Werner, and M. Hexamer, "Robust and self-tuning blood flow control during extracorporeal circulation in the presence of system parameter uncertainties," Medical and Biological Engineering and Computing, vol. 43, pp. 589-598, 2005.

[18] L.-X. Wang, "Stable adaptive fuzzy control of nonlinear systems," in Decision and Control, 1992., Proceedings of the 31st IEEE Conference on, 1992 , pp. $2511-2516$ vol.3.

[19] M. J. Er and Y. Gao, "Adaptive control strategy for blood pressure regulation using a fuzzy neural network," in Systems, Man and Cybernetics, 2003. IEEE International Conference on, vol. 3, oct. 2003, pp. 2120 2125 vol.3. 